\title{
Effect of Simultaneous Administration of Oral Polio Vaccine on Local Reaction of BCG Vaccine in Term Infants
}

\author{
MMA FARIDI AND SHITANSHU SRIVASTAVA \\ From Department of Pediatrics, Era's Lucknow Medical College, Lucknow, UP, India. \\ Correspondence to: Dr MMA Faridi, E-9 GTB Hospital, Delhi 110 095, India.drmmafaridi@gmail.com \\ Received: December 30, 2013; Initial review: February 06, 2014; Accepted: December 08, 2014.
}

Objective: To study local reaction and to ascertain timing of scar formation in infants after BCG vaccination at birth, with and without simultaneous administration of trivalent OPV.

Design: Prospective observational study.

Setting: Teaching hospital in Lucknow, India.

Participants: 152 term neonates born in the hospital and given BCG and OPV 0-dose simultaneously before discharge, within 7 days of birth (Group I), and 122 infants born at home or in private health facility, not given OPV-0 dose, coming for vaccination within 7 days of age (Group 2).

Intervention/Observation: Follow up done at 6 week, 10 week, 14 week and 9 months. Local reaction was recorded at the site of $B C G$ vaccination.

Results: Scar formed in $\leq 14$ wks in $51.3 \%$ and $89.3 \%$ babies in Group 1 and Group 2, respectively following BCG vaccination $(P<0.001)$. At 9 months, scar developed in 93.9\% infants in Group I and $94.3 \%$ babies in Group II. Abortive reaction and nonreactors were similar in both groups $(P>0.05)$.

Conclusions: Simultaneous administration of BCG vaccine with trivalent OPV to term infants in early neonatal period prolongs the time of scar formation but sequence of local reaction is not affected.

Keywords: Abortive reaction, BCG, Immunization, Neonate, OPV, Scar.
B CG is routinely administered to all newborn infants under the Universal Immunization Program. Oral Polio Vaccine (OPV) is recommended at birth together with BCG vaccine for all institutional deliveries [1]. Local reaction at the BCG vaccination site is described as papule, pustule, ulcer, scab, scar and abortive reaction; it may take 10 weeks to 6 months or more to form a scar [2]. Administering OPV together with BCG might downregulate the response to BCG vaccine [3]. The present study was planned to observe local reaction and time of scar formation in term appropriate-for-gestation age (AGA) infants, following BCG vaccination within 7 days of birth with or without simultaneous administration of trivalent OPV.

\section{Methods}

The present study was conducted in the Department of Pediatrics, Era's Medical College and Hospital, Lucknow, India during March 2008 to December 2010 after taking approval from the Institutional Ethics Committee and informed consent from parents. All consecutive term AGA babies [5] of both sexes born in the hospital with birth weight 2.2-4 kg were recruited. Consecutive neonates who were not delivered in the study hospital but came for vaccination within 7 days of birth were screened by history and available investigation reports done in the antenatal period, and were included if they fulfilled inclusion criteria. Infants having contact with tuberculosis patient or those born to mothers suffering from tuberculosis, severe anemia, diabetes, HIV/AIDS or Hepatitis B and those admitted in the neonatal intensive care unit for sepsis, intrauterine infection, jaundice, perinatal asphyxia, congenital malformation or suspected chromosomal anomalies, and family residing more than $10 \mathrm{~km}$ away from the hospital were excluded from the study.

The institution protocol is to discharge normal parturient mothers and their babies after 48 hours of birth; in case of Lower Segment Cesarean Section (LSCS) a mother is discharged on day 5-6 after removing abdominal stitches. BCG vaccine is given only on Thursdays; rest of the vaccines are administered on all working days. Therefore, BCG and OPV-0 dose cannot be administered simultaneously to all infants before discharge from the hospital. In home-delivered infants, the BCG vaccination is advised within 2 weeks but OPV 0 -dose is not recommended to them under National Immunization Schedule. The study subjects thus were divided in two groups: Group I: Infants born in the study hospital and received BCG and OPV 0-dose 
simultaneously within 7 days of age before discharge; and Group II: Babies born at home or in a private health facility, who did not receive either OPV-0 dose or Pulse Polio Program dose, and came to study hospital for immunization within 7 days of age. Only BCG vaccine was given to them.

All mothers were counseled for exclusive breastfeeding by a nurse or faculty trained in infant and young child feeding counseling at the time of discharge [6]. They were also counseled for regular follow-ups and immunization on subsequent visits as per National Immunization Schedule.

One trained staff nurse with more than 5 years of experience in the immunization clinic administered 0.1 $\mathrm{mL}$ of BCG vaccine (Danish 1331 strain; BCG laboratory, Guindy Chennai) to all enrolled babies. The freeze-dried vaccine was reconstituted with normal saline and given intradermal on the left arm just above the insertion of the deltoid muscle with a 26-gauge needle and tuberculin syringe to produce a wheal of 5 $\mathrm{mm}$. The reconstituted vaccine was used within 4 hours and then discarded. Cold chain was maintained and monitored as per standard protocol. Oral trivalent polio vaccine Sabin strain (Bharat Biotech) was administered to babies in Group I simultaneously with BCG. OPV was not administered to infants belonging to Group II. Cold chain was maintained and vaccine viability was checked with the help of vaccine vial monitor.

Four follow-ups were done at $6(+1)$ week, $10(+1)$ week, 14 ( +1 week) and 9 months ( +2 weeks). The local reaction at the vaccination site was recorded as papule, pustule, ulcer, scab, scar, no reaction, and abortive reaction [2]. Babies who did not have any reaction at the inoculation site at 14 weeks were labeled as non-reactor. Infants who did not develop scar by 14 weeks but had papule, pustule or ulcer were counseled for follow-up at 9 months. Local reaction was recorded by a single observer in all subjects. If there was any ambiguity, both authors concurred for abortive reaction or no reaction.

Statistical analyses: The data were entered in Microsoft Excel and checked for any inconsistency. Univariate logistic regression analysis was carried out to find out any relationship of scar formation at $6,10,14$ weeks and 9 months with sex and birth weight of the infants. $P$ value $<0.05$ was considered as significant. Analysis was carried out with SPSS Version 16.0.

\section{RESUlts}

Out of 275 babies recruited (Fig. 1), Group I comprised of 152 consecutive neonates. Four of these infants were lost to follow-up and 148 infants were included for analysis. Group II comprised of 123 neonates, who received only BCG. One child was lost to follow-up due to change of residence as per telephonic conversation. Hence, data of 122 infants were analyzed.

Number of infants delivered normally, with the help of forceps and by LSCS, socioeconomic status of the parents and clinical profile of the mothers such as hemoglobin level, parity and antenatal check-up status were comparable in both groups. The mean (SD) birth weight of babies in Group I [2.73(0.30 kg)] and Group II [2.75 $(0.32 \mathrm{~kg})]$ was comparable $(P=0.57)$. The mean age of infants at immunization was 2.6 days (range 1-6 days) and 3.9 days (range 2-7 days) in Group I and II, respectively. Both groups were sex- and age-matched. Sex and birth weight of the infants did not affect either the development of local reaction or time of scar formation in both groups.

The advent and sequence of local reaction was similar in both groups at 6,10 and 14 weeks following BCG vaccination (Table I). A higher $(P<0.01)$ proportion of infants deceloped scar at 6 weeks in Group II infants (25.4\%), in comparison to infants of Group I (3.4\%).

Table II depicts the comparison of scar formation between infants of Group I and II. The development of

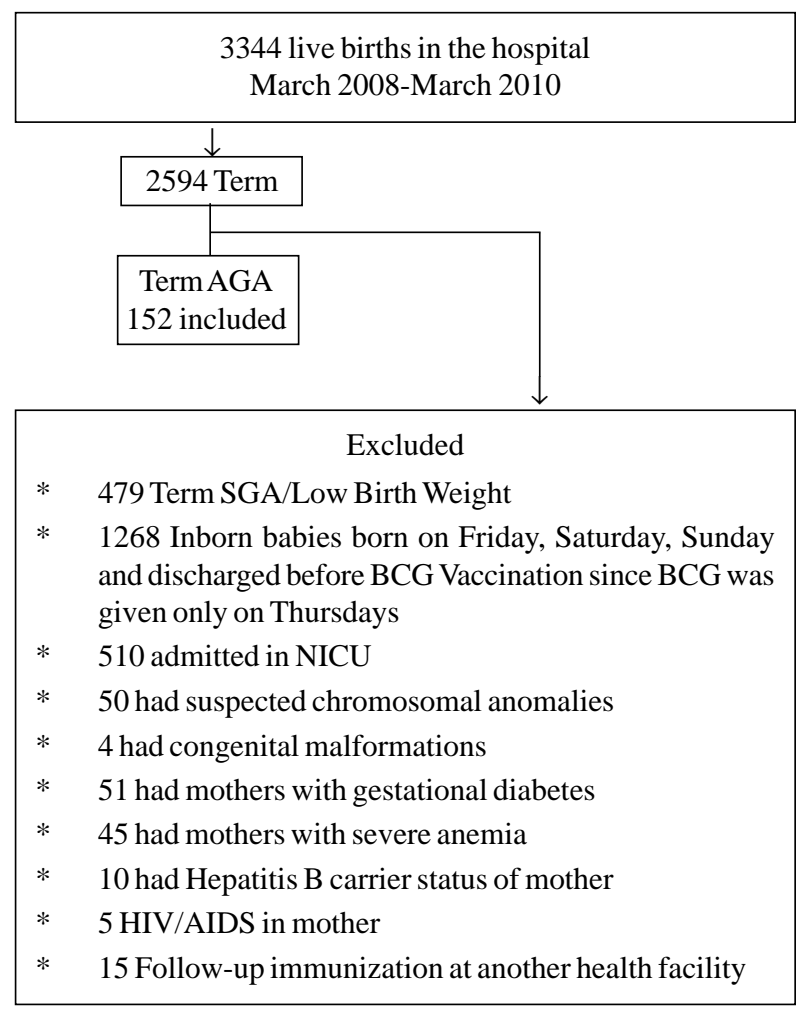

FIG. 1 Recruitment flow of participants

VOLUMe 52-FEBRUARY 15, 2015 
scar was significantly lower among Group I as compared to Group II infants till 14 weeks post-vaccination. The cutaneous reaction at the site of BCG immunization in the form of either scar or abortive reaction was similar (96.6\% vs $96.8 \%$ ) in both groups.

\section{Discussion}

Our study demonstrated that simultaneous administration of OPV with BCG vaccine at birth may affect local BCG reaction. The sequence of cutaneous reaction, incidence of abortive reaction and number of non-reactors were similar in infants given BCG and OPV together and those who received BCG vaccine alone. However, scar formation lagged behind at 6, 10, and 14 weeks post-immunization in infants who received both BCG and OPV together. Eventually scar formed by 9 months in all infants at the site of BCG inoculation.

Interaction between the two live vaccines, BCG and OPV, administered simultaneously at birth, was studied by the development of local cutaneous reaction but effect on in vivo immune response was not studied by us. The other limitations were non-randomized design of study, and no blinding of investigators.
OPV is widely administered along with BCG at birth in developing countries but little is known regarding the adverse impact on cellular immune responses following vaccination [4]. The development of scar at the site of BCG vaccination is regarded as an evidence of successful vaccination $[3,7-8]$ and may be taken as a surrogate marker for development of immunogenicity $[9,10]$. It has been reported that the scar forms in $65.3 \%$ to $100 \%$ infants following BCG vaccination (7-14). In all these studies, OPV was not administered simultaneously with BCG vaccine. Kaur, et al [3]. opined that BCG and OPV given together at birth might delay scar formation by 3 months of age [3]. Sartono, et al. [4] demonstrated that mucosal administration of OPV simultaneously with intradermal BCG vaccination at birth may have profound immunological consequences. They reported that this may down- regulate response to BCG in the form of fewer numbers of scar formation, reduced scar size, reduced in vivo response to purified protein derivative (PPD) and significantly lower IL-13 and IFN- $\gamma$ and a tendency to have lower IL-10 in response to PPD at 6 weeks following immunization [4]. Jensen, et al. [15] in a recent study have also shown that giving OPV and BCG together to neonates led to

TABLE I LOCAL BCG REACTION IN GROUP I (BCG AND OPV0 DOSE) AND GROUP II (ONLY BCG) INFANTS

\begin{tabular}{|c|c|c|c|c|c|c|c|c|}
\hline \multirow[t]{2}{*}{ Local Reaction } & \multicolumn{2}{|c|}{$\begin{array}{l}6 \text { wks } \\
\text { Group }\end{array}$} & \multicolumn{2}{|c|}{$\begin{array}{l}10 \text { wks } \\
\text { Group }\end{array}$} & \multicolumn{2}{|c|}{$\begin{array}{l}14 \text { wks } \\
\text { Group }\end{array}$} & \multicolumn{2}{|c|}{$\begin{array}{l}9 \text { months } \\
\text { Group }\end{array}$} \\
\hline & INo. (\%) & II No. (\%) & INo. (\%) & II No. (\%) & INo.(\%) & II No. (\%) & INo. (\%) & II No. (\%) \\
\hline Induration & 30 (20.3) & $5 \quad(4.1)$ & 0 & 0 & 0 & 0 & 0 & 0 \\
\hline Papule & 54 (36.5) & 13 (10.7) & 14 (9.5) & $5 \quad(4.1)$ & 0 & 0 & 0 & 0 \\
\hline Pustule & $39(26.4)$ & $28(22.9)$ & 43 (29.1) & $3 \quad(2.5)$ & 10 (6.8) & $1(0.8)$ & 0 & 0 \\
\hline Ulcer & 10 (6.8) & $33(27.0)$ & 30 (20.3) & $18(14.8)$ & 20 (13.5) & $4(3.3)$ & 0 & 0 \\
\hline Scab & $0 \quad(0.0)$ & $7 \quad(5.7)$ & 35 (23.6) & $2(1.6)$ & 33 (22.3) & $1(0.8)$ & 0 & 0 \\
\hline No reaction & 10 & $5 \quad(4.1)$ & $8 \quad(5.4)$ & $4 \quad(3.3)$ & $5 \quad(3.4)$ & $4(3.3)$ & $5(3.4)$ & $4(3.3)$ \\
\hline Abortive reaction & & & & & $4 \quad(2.7)$ & $3(2.5)$ & $4(2.7)$ & $3(2.5)$ \\
\hline
\end{tabular}

$N=148$ in Group I and 122 in Group II.

TABLE II COMPARISON OF SCAR FORMATION IN TWO GROUPS

\begin{tabular}{|c|c|c|c|c|c|c|c|c|}
\hline \multirow[t]{2}{*}{ Reaction } & \multicolumn{2}{|c|}{$\begin{array}{l}6 \text { wks } \\
\text { Group }\end{array}$} & \multicolumn{2}{|c|}{$\begin{array}{l}10 \text { wks } \\
\text { Group }\end{array}$} & \multicolumn{2}{|c|}{$\begin{array}{l}14 \text { wks } \\
\text { Group }\end{array}$} & \multicolumn{2}{|c|}{$\begin{array}{l}9 \text { months } \\
\text { Group }\end{array}$} \\
\hline & INo. (\%) & II No. (\%) & INo. (\%) & II No. (\%) & INo. (\%) & II No. (\%) & INo. (\%) & II No. (\%) \\
\hline Scar & $5 \quad(3.4)$ & $31(25.4)$ & $18(12.1)$ & $90(73.7)$ & $76(51.3)$ & 109 (89.3) & $139(93.9)$ & 115 (94.3) \\
\hline No scar & 143 (96.6) & $91(74.6)$ & $130(87.8)$ & $32(26.2)$ & $72(48.6)$ & 13 (10.7) & $(6.1)$ & $7 \quad(5.7)$ \\
\hline OR (95\% CI), & \multicolumn{2}{|c|}{$0.10(0.04-0.27)$} & \multicolumn{2}{|c|}{$0.05(0.03-0.09)$} & \multicolumn{2}{|c|}{$0.13(0.07-0.24)$} & \multicolumn{2}{|c|}{$0.94(0.34-2.60)$} \\
\hline$P$ value & \multicolumn{2}{|c|}{$<0.001$} & \multicolumn{2}{|c|}{$<0.001$} & \multicolumn{2}{|c|}{$<0.001$} & \multicolumn{2}{|c|}{0.91} \\
\hline
\end{tabular}

${ }^{*}$ Derived by univariate logistic regressionN $=148$ in Group I and 122 in Group II. 


\section{What Is AlReady Known?}

- The formation of scar at the site of BCG vaccination is regarded as an evidence of successful immunization.

WhAT ThIS StUdY AdDS?

- Simultaneous administration of BCG and OPV at birth prolongs the time of scar formation.

significantly lower prevalence of IFN- $\gamma$ and reduced levels of interleukin-5 to PPD, implying that OPV affected both Th-1 and Th-2 cytokine responses. Most BCG efficacy studies were done prior to the introduction of OPV at birth. It may be speculated that the addition of OPV to BCG at birth has further compromised the protection induced by BCG [4]. After BCG vaccination, a cascade of reaction takes place where IL-2, interferon$\gamma$ and TNF- $\alpha$ are predominantly secreted. Inflammation and suppuration at the site of BCG vaccination are mediated through interleukins [16]. Which of these reaction/s or cascade is affected by OPV, if administered simultaneously, at the time of BCG vaccination is an area of research.

We conclude that simultaneous administration of OPV with BCG vaccine delays scar formation, but it does not affect sequential development of the local reaction or proportion of non-reactors.

Contributors: Both authors participated in study design, data collection and analysis, and manuscript writing.

Funding; None; Competing interests: None stated.

\section{REFERENCES}

1. Yewale V, Choudhary P, Thackar N, editors. IAP Guide Book on Immunization. IAP Committee on Immunization 2009-2011. p. 47-52.

2. Faridi MMA, Krishnamurthy S. Abortive reaction and time of scar formation after BCG vaccination. Vaccine. 2008;17:26:289-90.

3. Kaur S, Faridi MMA, Agarwal KN. BCG vaccination reaction in low birth weight infants. Indian J Med Res. 2002;116:64-9.

4. Sartono E, Lisse IM, Terveer EM, Van de Sande PJM, Whittle H, Fisker AB, et al. Oral polio vaccine influences the immune response to BCG vaccination.
PLoS One. 2010;5: e10328.

5. Lubchenco LO, Hansman C, Dressler M, Boyd E. Intrauterine growth as estimated from live born birth weight data at 24 to 42 weeks of gestation. Pediatrics. 1963;32:793-800.

6. Infant and Young Child Feeding Counseling: A Training Course: The 3 in 1 course-2008 Version (An Integrated Course on Breastfeeding, Complementary feeding and Infant Feeding \& HIV - Counseling). Breastfeeding Promotion Network of India and IBFAN Asia.

7. Seth V, Kabra SK. BCG Vaccination. In: Essentials of Tuberculosis in Children, 3rd Ed; 2006; p. 597-620.

8. Thayyil-sudan S, Kumar A, Singh M, Paul VK, Deorari AK. Safety and efficacy of BCG vaccination in preterm babies. Arch Dis Child. 1999;81:F-646.

9. Gupta P, Faridi MMA, Shah D, Dev G. BCG reaction in twin newborns: Effect of zygosity and chorionicity. Indian Pediatr. 2008;45:271-7.

10. Ahmad S, Javid I. Absence of scar formation in infants after BCG vaccination. Professional Med J. 2006;13:637-41.

11. Lakhar BB. Neonatal BCG vaccination and scar success. Indian Pediatr. 1995; 32:1323.

12. Joshi S. BCG vaccination (immunization dialogue). Indian Pediatr. 2000;37:332-3.

13. Sedaghatian M, Hashaem F, Hossain MM. Bacillus Calmette-Guerin vaccination in preterm infants. Int $\mathrm{J}$ Tuber Lung Dis.1998;2:679-82.

14. Fine PEM, Ponnighaus JM, Maine N. The distribution and implication of BCG scar in Northern Malawi. Bull World Health Organ. 1989;67:35-42.

15. Jensen KJ, Karkov HS, Lund N, Andersen A, Eriksen HB, Barbosa AG, et al. The immunological effects of oral polio vaccine provided with BCG vaccine at birth: A randomised trial. Vaccine. 2014;32:5949-56.

16. Sallusto F, Lenig D, Förster R, Lipp M, Lanzavecchia A.Two subsets of memory $\mathrm{T}$ lymphocytes with distinct homing potentials and effector functions. Nature. 1999;401:708-12. 